\title{
Quantificação da ecogenicidade da doença pancreática gordurosa não alcoólica - esteatometria pancreática
}

\author{
Quantification of ecogenicity of non-alcoholic fatty pancreatic disease - pancreatic \\ steatometry
}

\author{
Luís Jesuíno de Oliveira Andrade ${ }^{1 *}$, Gabriela Correia Matos de Oliveira ${ }^{2}$ \\ ${ }^{1}$ Doutor, Professor Titular do Departamento de Ciências da Saúde - Universidade Estadual de Santa Cruz - Ilhéus - Bahia \\ - Brasil; ' 2 Acadêmica de Medicina - Faculdade de Medicina - UniFTC - Salvador - Bahia - Brasil.
}

\begin{abstract}
Resumo
Introdução: a ultrassonografia tem sido utilizada na identificação da doença pancreática gordurosa não alcoólica (DPGNA), entretanto o método apresenta como desvantagem a subjetividade do examinador. Objetivo: quantificar a ecogenicidade pancreática para classificar em graus a DPGNA. Metodologia: utilizou-se a análise de histograma de escala de cinzas para quantificar a ecogenicidade, com a finalidade de análise mais acurada das características do tecido pancreático. Inicialmente, foi calculado o intervalo representativo da ecogenicidade pancreática e renal (média \pm 2 desvios-padrão, que corresponde aos $97,5 \%$ mais representativos do tecido). Posteriormente, foi determinado o percentual de sobreposição da ecogenicidade do pâncreas com o rim analisado. Resultados: consideraram-se como normal quando o percentual de sobreposição de ecogenicidade estava em até $25 \%$; esteatose grau I para percentual entre 25 e $50 \%$; esteatose grau II para percentual entre 50 e $75 \%$; esteatose grau III para percentual acima de $75 \%$.Conclusão: os autores concluem que, com quantificação de ecogenicidade, a classificação de DPGNA torna-se não examinador-dependente.

Palavras-chaves: Ultrassonografia. Pâncreas. Gorduras. Diagnóstico por imagem.
\end{abstract}

\begin{abstract}
Ultrasonography has been used in the identification of non-alcoholic fatty pancreatic disease (NAFPD) however the method presents the subjectivity of examiner as disadvantage. Objective: the aim of this study is to quantify pancreas echogenicity and classify NAFPD degrees. Methodology: to provide for an accurate analysis, a gray-scale histogram was used to quantify the liver parenchyma echogenicity. Initially, a calculation was made to find the representative interval of liver and underlying kidney echogenicity (average \pm 2 standard deviations, which corresponds to the $97.5 \%$ of the most representative portion of the tissue). Afterwards, the percentage of overlapping echogenicity of kidney was determined. Results: results were considered normal when the percentage of overlapping echogenicity was up to 25\%; mild steatosis (or degree 1), a percentage between 25\% and 50\%; moderate steatosis (degree 2), a percentage between $50 \%$ and $75 \%$; and severe steatosis (degree 3 ), a percentage above $75 \%$. Conclusion: the quantification and classification of pancreas echogenicity for NAFPD using a gray-scale histogram is objective and non-examiner dependent.

Keywords: Ultrasonography. Pancreas. Fats. Diagnostic imaging.
\end{abstract}

\section{NTRODUÇÃO}

A infiltração gordurosa do pâncreas foi relatada pela primeira vez por Ogilvie em $1993^{1}$, denominada de doença pancreática gordurosa não alcoólica (DPGNA), pâncreas gorduroso, lipomatose pancreática, ou esteatose pancreática.

O diagnóstico dessa condição depende principalmente de procedimentos por imagem como ressonância magnética, tomografia computadorizada ou ultrassonografia ${ }^{2}$. O estudo histológico obtido por biópsia pancreática é considerado como padrão "ouro" para o diagnóstico de esteatose pancreática, porém, sua indicação é limitada a casos selecionados, sendo a ultrassonografia uma opção para identificar a DPGNA, mas que apresenta como desvantagem a subjetividade do examinador.

Correpondente/Corresponding: *Luís Jesuíno de Oliveira Andrade-End: Departamento de Ciências da SaúdeUniversidade Estadual de Santa Cruz - UESC - CEP 45662-900. Ilhéus-Bahia, Brasil. - E-mail: luis_jesuino@ yahoo.com.br - Tel: +5573988122755
Os critérios ecográficos para o diagnóstico de DPGNA consistem em: aumento de fundo da ecogenicidade em relação à ecogenicidade renal e ao retroperitônio, atenuação do feixe acústico e áreas focais de maior ecogenicidade ${ }^{3}$.

O objetivo deste estudo foi quantificar a ecogenicidade pancreática, buscando definir em grau a infiltração gordurosa, classificando-se a DPGNA de forma objetiva, portanto, não examinador-dependente. Trata-se de um estudo descritivo, utilizando-se a análise de histograma de escala de cinzas, que quantifica a ecogenicidade do tecido pancreático de forma mais acurada, tendo em vista que a padronização dos resultados nos diversos equipamentos não é recomendável, em função das variações conforme o contraste e brilho ajustados no aparelho de ultrassonografia.

\section{METODOLOGIA}

Foram selecionadas retrospectivamente 100 imagens ultrassonográficas do pâncreas de 100 diferentes indivíduos adultos dos sexos masculino e feminino, assim 
distribuídas: 25 imagens consideradas como normais, 25 imagens DPGNA grau I, 25 imagens DPGNA grau II e 25 imagens como DPGNA grau III.

Por trata-se de um estudo com bando de dados de imagens, não envolvendo pesquisa com seres humanos, não foi submetido ao Comitê de Ética em Pesquisa com Seres Humanos. Entretanto, todos os preceitos éticos necessários para a análise e divulgação dos dados da pesquisa foram seguidos.

O diagnóstico ultrassonográfico (US) de DPGNA ocorreu incidentalmente, tendo em vista que os exames realizados foram solicitados devido a queixas não relacionadas com DPGNA. Para a análise das imagens foi utilizado o software Adobe Photoshop $\mathrm{CC}^{\circ}$ que permite a análise de histogramas de imagens, pois consegue identificar 32.768 tons de cinzas (Figura 1).

Figura 1 - Histograma da escala de cinzas

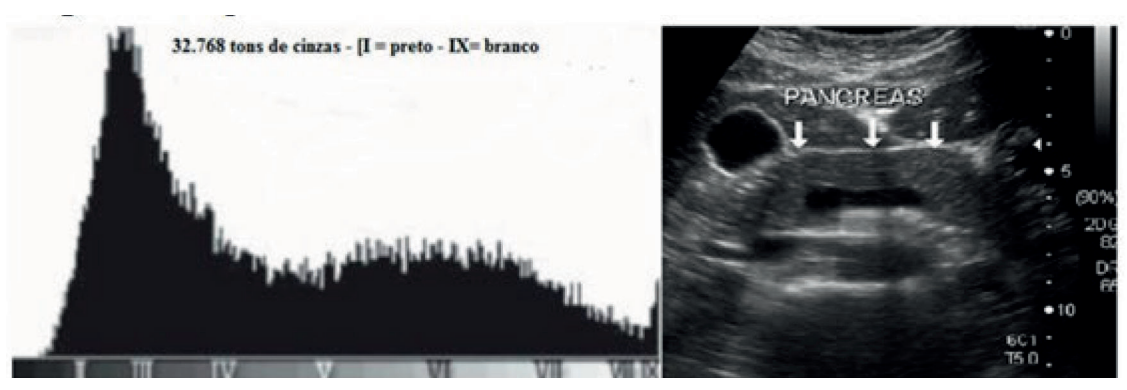

Fonte: Acervo do autor, 2019.

Inicialmente, foi calculado o intervalo representativo da ecogenicidade pancreática e do rim (média \pm 2 desvios-padrão), que corresponde aos $97,5 \%$ mais representativos do tecido, segundo os critérios já bem definidos da ecogenicidade de ambos os órgãos. Em seguida determinou-se a percentagem de sobreposição da hiperecogenicidade do pâncreas com o rim analisado.

A partir da sobreposição das ecogenicidades pancreática e renal, foram estabelecidos os percentuais para classificação em graus da esteatose pancreática em leve (Grau I - levemente hiperecogênico), moderada (Grau II - moderadamente hiperecogênico) e grave (Grau III intensamente hiperecogênico).

\section{Aquisição das imagens ecográficas}

As aquisições das imagens analisadas foram obtidas por um único e experiente médico especialista em ultrassonografia, para minimizar viés de diferentes examinadores, realizadas no mesmo equipamento de alta resolução (Toshiba Xario XG) usando uma sonda convexa de $3,5 \mathrm{MHz}$. O ganho geral do aparelho ajustado em $50 \%$, permitindo, assim, maior flexibilidade no ajuste da curva de ganho para obter ecogenicidade uniforme em todas as profundidades das imagens. As imagens ultrassonográficas foram adquiridas do acervo de imagens de uma clínica de gastrenterologia da cidade de Itabuna - Bahia. Os pacientes foram examinados em decúbito dorsal, traçando-se a região do pâncreas cobrindo todo o órgão ao nível da artéria mesentérica superior. Foi realizado também corte ecográfico no plano longitudinal ao longo de eixo maior do rim direito, obtendo-se uma imagem do lobo direito hepático com o rim direito adjacente (Figura 2).

Figura 2 - Plano de corte para aquisição do histograma

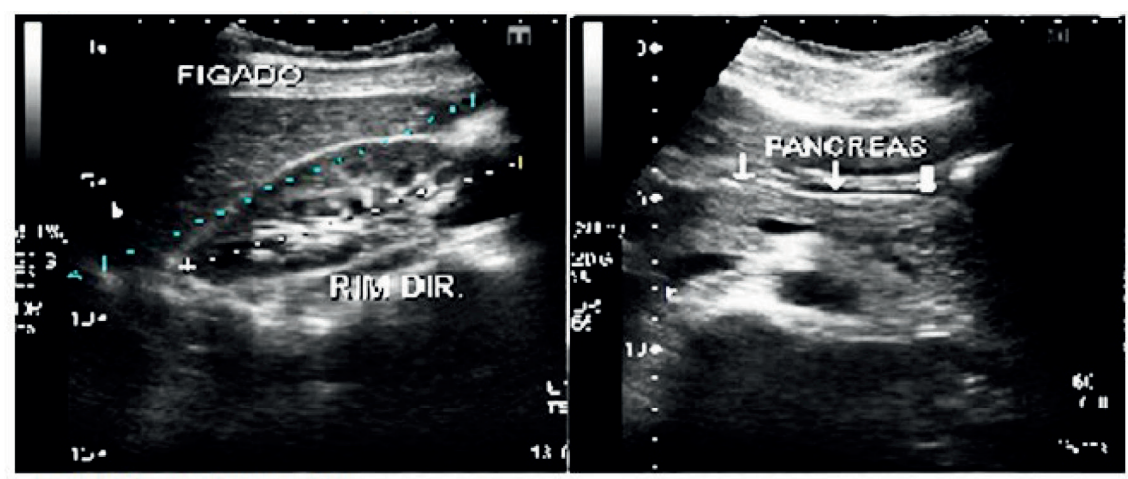

Fonte: Acervo do autor, 2019.

A DPGNA foi diagnosticada através do aumento da comparação realizada em janelas ecográficas diferentes. ecogenicidade do pâncreas em relação ao rim, sendo esta 


\section{Quantificação da ecogenicidade na DPGNA}

Para a quantificação da ecogenicidade pancreática e renal, a imagens a serem analisadas foram exportadas para o formato digital DICOM (Digital Imaging and Communications in Medicine) e transportadas para o desktop do computador para análise. Para avaliação do histograma das imagens foi utilizado o software Adobe Photoshop CC ${ }^{\circ}$, analisando-se a partir daí um intervalo representativo do pâncreas e o córtex renal direito (Figura 3).

Figura 3 - Intervalos representativos analisados

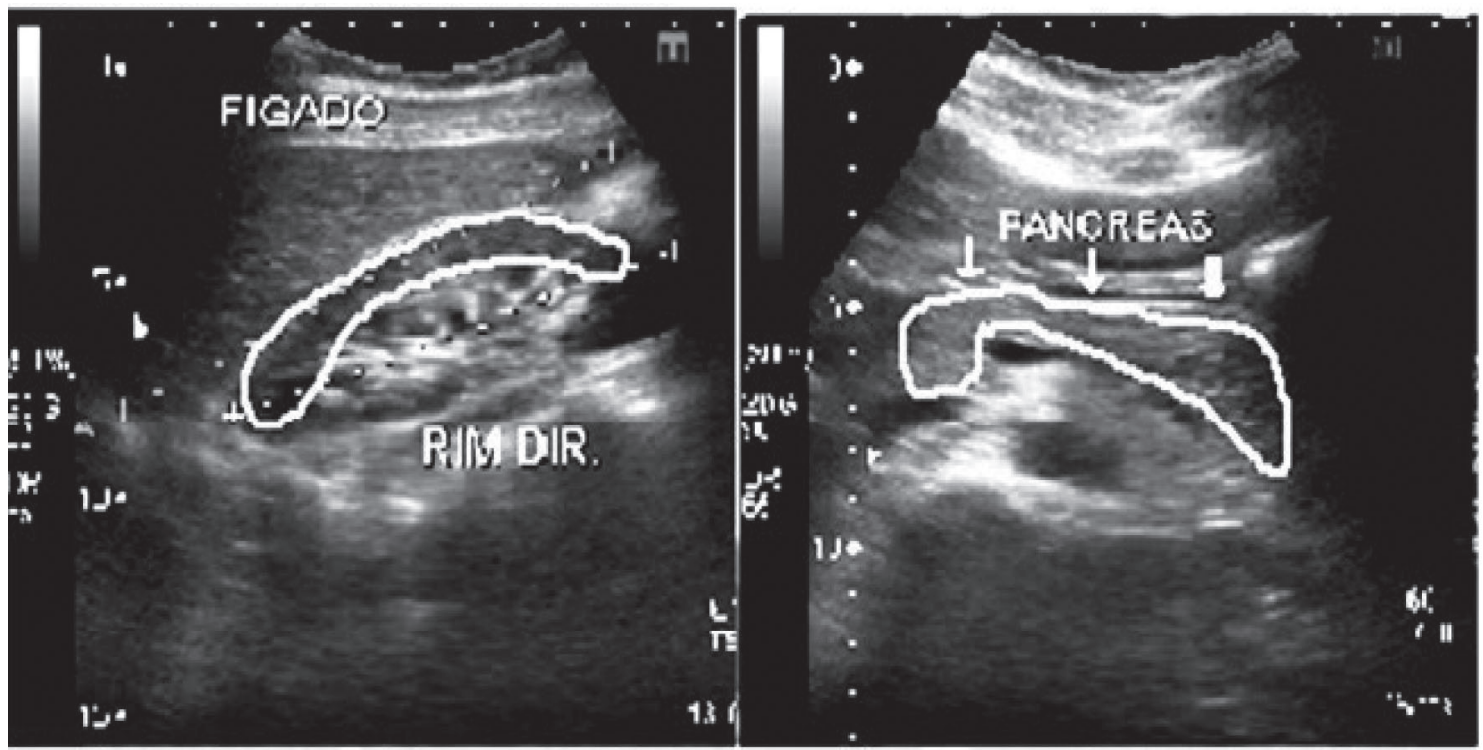

Fonte: Acervo do autor, 2019.

Considerou-se como normal quando o percentual de sobreposição da ecogenicidade pancreática em relação ao rim direito estava em até $25 \%$ (Figura 4).

Figura 4 - Pâncreas normal.

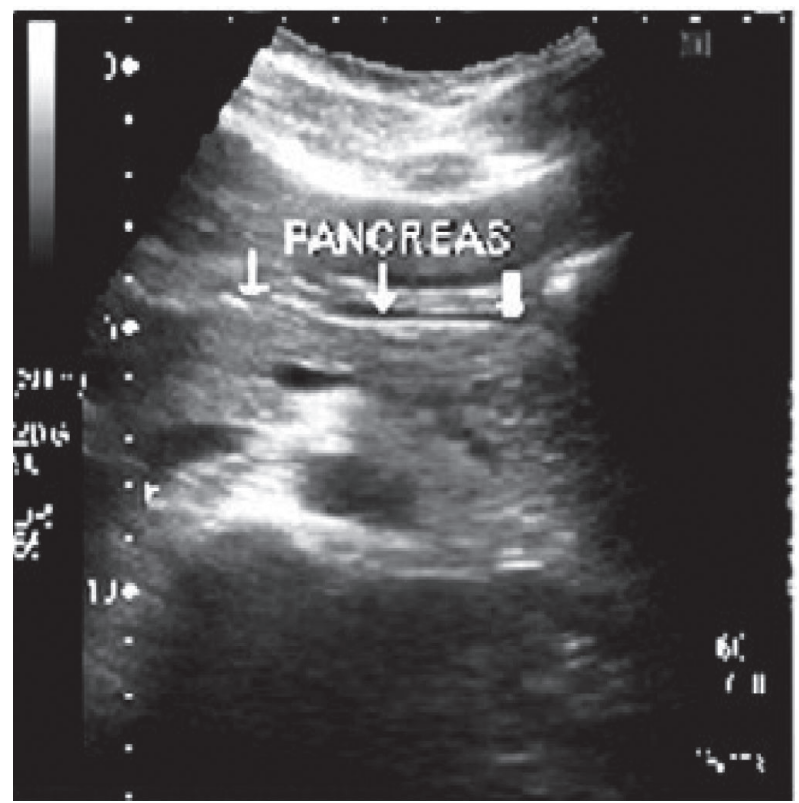

Fonte: Acervo do autor, 2019.

A análise do histograma do pâncreas normal está representada na figura 5 .
Figura 5 - Histograma do pâncreas normal.

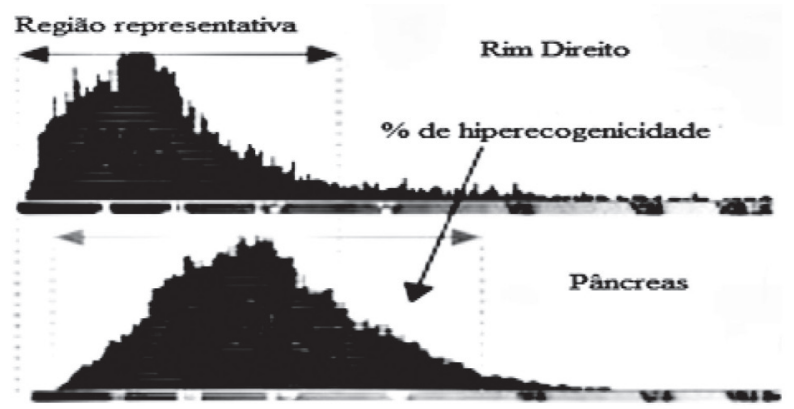

Fonte: Resultado do estudo

Considerou-se como DPGNA grau I (levemente hiperecogênico) para um percentual de sobreposição da hiperecogenicidade pancreática, em relação ao rim direito, entre 25 e $50 \%$ (Figura 6).

Figura 6 - Esteatose pancreática grau I. 


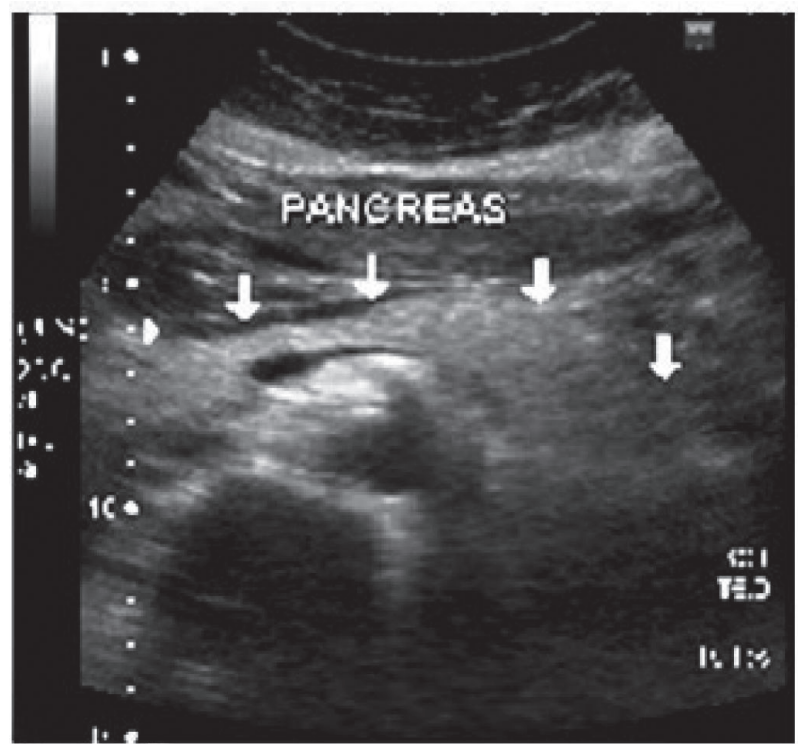

Fonte: Acervo do autor, 2019

A análise do histograma do pâncreas com DPGNA grau I está representada na figura 7.

Figura 7 - Histograma de pâncreas com esteatose grau I.

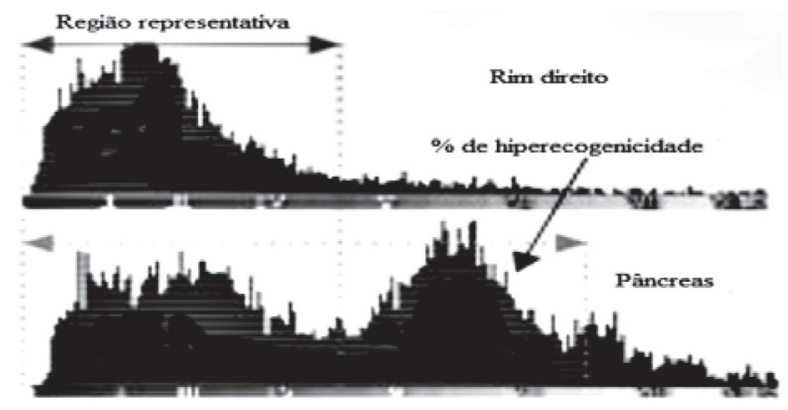

Fonte: Resultado do estudo

Considerou-se como DPGNA grau II (moderadamente hiperecogênico) para uma percentagem de sobreposição da hiperecogenicidade pancreática, em relação ao rim direito, entre 50 e $75 \%$ (Figura 8).
Figura 8 - Esteatose pancreática grau II.

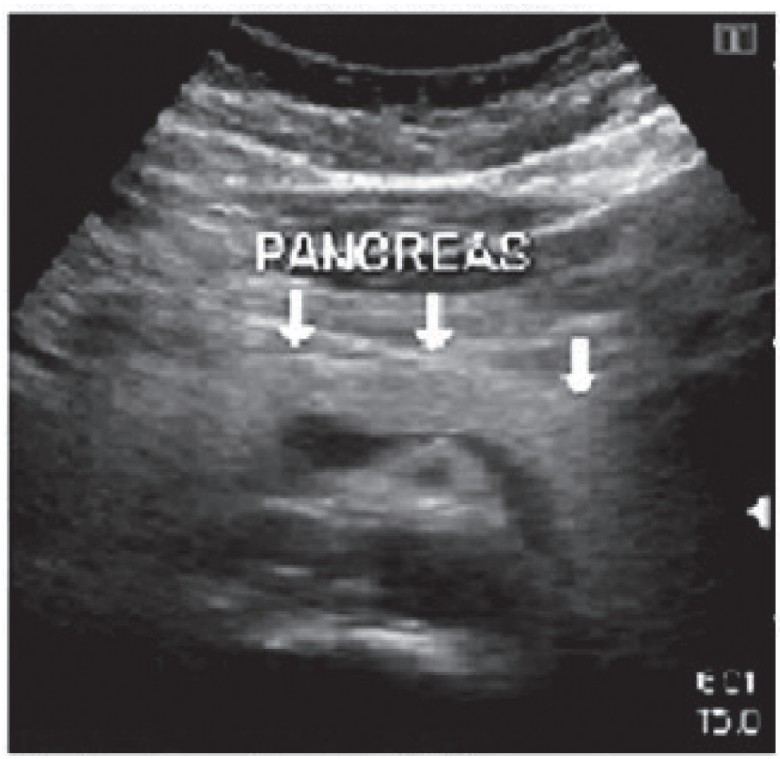

Fonte: Acervo do autor, 2019

A análise do histograma do pâncreas com DPGNA grau Il está representada na figura 9.

Figura 9 - Histograma do pâncreas com esteatose grau II.

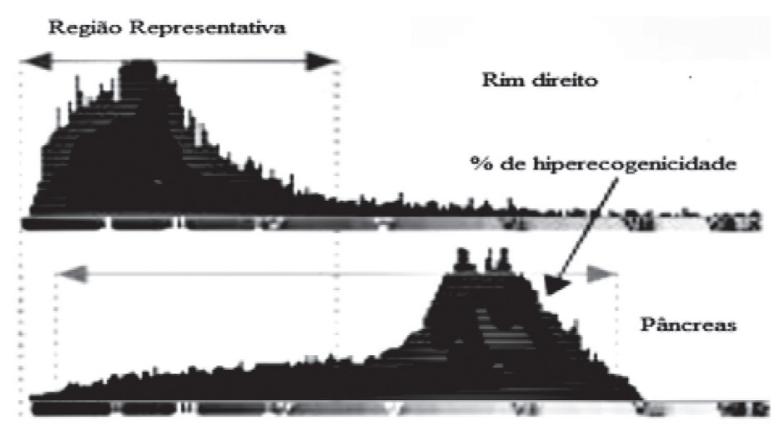

Fonte: Resultado do estudo

Considerou-se como DPGNA grau III (intensamente hiperecogênico) para um percentual de sobreposição da hiperecogenicidade pancreática, em relação ao rim direito, acima de $75 \%$ (Figura 10). 
Figura 10 - Esteatose pancreática grau III.



Fonte: Acervo do autor, 2019

A análise do histograma do pâncreas com DPGNA grau III está representada na figura 11.

Figura 11 - Histograma do pâncreas com esteatose grau III.

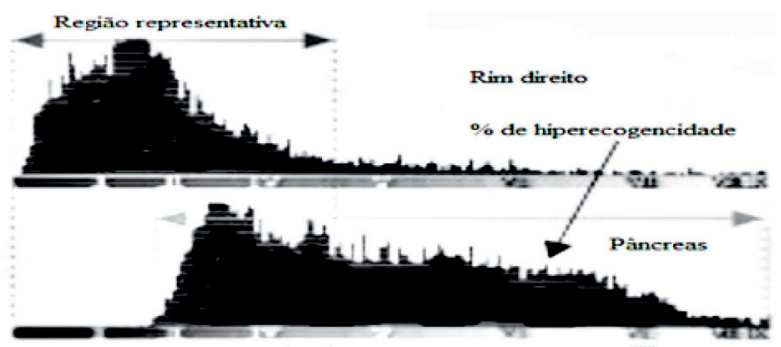

Fonte: Resultado do estudo

\section{DISCUSSÃO}

A atenuação do feixe acústico US foi estimada neste trabalho, por meio da quantificação da ecogenicidade, determinando o grau de esteatose do parênquima pancreático.

O exame US pode ser de valor limitado na avaliação de todo o pâncreas não só pela sua localização retroperitonial, como também por informações quantitativas às vezes não confiáveis ${ }^{4}$, levando-se ainda em consideração que o teor da gordura pancreática, tem que alcançar um percentual acima de $4 \%$ para ser detectado ${ }^{5}$. A subjetividade na avaliação da quantidade de gordura necessária para a determinação dos graus de DPGNA tem sido relatada como uma limitação potencial do uso da ultrassonografia modo $B$ na prática clínica ${ }^{6}$. Neste estudo demonstrou-se que a esteatometria é uma alternativa objetiva para o diagnóstico de DPGNA, minimizando assim a subjetividade na avaliação quantitativa da gordura necessária para de- finição dos graus da DPGNA e, portanto sugerindo, que a incapacidade de quantificar a ecogenicidade do pâncreas deixa de ser uma limitação diagnóstica.

O estudo US tem sido utilizado na avaliação das doenças do pâncreas em função de ser um método sensível na diferenciação entre o normal e o patológico, além da vantagem de ser um exame inócuo, não invasivo e de baixo custo. Além disso, a ultrassonografia é o exame de imagem mais acessível para o diagnóstico de fibrose e esteatose, embora a sensibilidade varie de 37 a $94 \%$ e especificidade de 48 a $100 \%{ }^{7}$.

Estudos demonstraram que a frequência de DPGNA, em indivíduos que foram avaliados por ultrassonografia, variou de $27,8-46,0 \%{ }^{8,9}$, enquanto que com ressonância magnética foi de $16,1 \%$, relatada em um estudo chinês ${ }^{10}$. Alguns pesquisadores acreditam que a DPGNA ou a deposição de gordura nas células pancreáticas não existe em humanos ${ }^{11}$, porém outras observações demonstraram que a deposição de gordura ocorre principalmente em septos interlobulares ${ }^{12}$

Existe uma associação bem documentada entre idade avançada e DPGNA ${ }^{13}$. Um relato encontrou que a deposição de gordura foi significativamente aumentada nas ilhotas pancreáticas em indivíduos acima de 60 anos $^{14}$. A avaliação com ultrassonografia endoscópica e estudos de autópsia reforça esta associação ${ }^{15}$.

Do ponto de vista ultrassonográfico, a DPGNA é classificada em três graus: Grau I = DPGNA leve, em que a ecogenicidade pancreática é levemente maior que a ecogencidade renal; Grau II = DPGNA moderada, com ecogenicidade do pâncreas moderadamente maior que a ecogenicidade renal; e Grau III = DPGNA severa, em que a ecogenicidade pancreática é muito superior a ecogenicidade do rim $^{16}$.

Apesar da sensibilidade e especificidade elevadas ao exame ultrasonográfico na identificação da infiltração gordurosa pancreática, a subjetividade do examinador é ainda um fator limitante. Neste estudo, utilizando-se a análise de histograma de escala de cinzas, a ecogenicidade da imagem do pâncreas foi quantificada e com isso, determinado percentuais de ecogenicidade, que quantificam o grau de infiltração gordurosa, tornando o método ultrassonográfico no diagnóstico da DPGNA objetivo e não examinador-dependente.

O histograma revela a distribuição dos níveis de cinza da imagem. É representado por um gráfico, indicando o número de pixels na imagem para cada nível de cinza, sumarizando uma imagem através de seus tons de cinza. Informa o número ou a proporção de pixels que possuem um determinado nível de cinza.

Visando padronizar os resultados nos diversos equipamentos de ultrassonografia, não é recomendado o uso do valor puro da ecogenicidade, frente à suscetibilidade das variações conforme o ganho e brilho ajustados no aparelho. Ao utilizar-se o histograma comparando o tecido pancreático com o tecido renal e fazendo a classificação da DPGNA de acordo com os dados encontrados, elimina-se, 
portanto, as variações anteriormente citadas.

Os autores utilizaram o software Adobe Photoshop $\mathrm{CC}^{\circledast}$ que permite a análise de histogramas de imagens, pois consegue identificar 32.768 tons de cinzas; entretanto, outros programas de análise de imagem que possuem o recurso de histograma podem ser utilizados.

A literatura médica não apresenta pesquisas sobre o uso de imagens ultrassonográficas do pâncreas, para avaliação da esteatose utilizando histogramas. Trabalho recente examinou 60 crianças hospitalizadas, avaliando-se ecograficamente a presença e grau de DPGNA, usando comparação pareada de ecogenicidade pancreática com ecogenicidade renal e retroperitoneal, porém utilizaram o coeficiente de atenuação do ultrassom ${ }^{17}$. Outros autores avaliaram o depósito de gordura pancreática através de histograma com limiarização local e a medida da atenuação do parênquima estimado a partir de valores de unidades HU de tomografia computadorizada ${ }^{18}$.

DPGNA é considerada a doença pancreática mais prevalente em todo mundo ${ }^{19}$, especialmente em função da alta incidência de patologias como obesidade, dislipidemia e diabetes. Frente a tais dados este estudo pretende contribuir para o diagnóstico US mais preciso e objetivo.

Os autores concluem que com uma quantificação de ecogenicidade a classificação da DPGNA, torna-se objetiva, portanto, não examinador-dependente.

Mais estudos devem ser realizados, utilizando o método de histograma de imagens, correlacionando com outros métodos diagnósticos através do desenvolvimento de software de computador, para fornecer dados quantitativos adicionais, gerando histogramas, que podem permitir medir e quantificar o número de células gordurosas no pâncreas ou outras vísceras parenquimatosas através da computação cognitiva.

\section{AGRADECIMENTOS}

Agradecemos a Dra. Célia Kalil Mangabeira pela revisão gramatical do manuscrito.

\section{REFERÊNCIAS}

1. OGILVIE, R. F. The islands of Langerhans in 19 cases of obesity. J. Pathol., England, v.7, n. 37, p. 473-481, 1933.

2. TUSHUIZEN, M. E. et al. Pancreatic fat content and beta-cell function in men with and without type 2 diabetes. Diabetes Care., New York, v. 30, n. 11, p. 2916-2921, 2007.

3. YANG, D.M. et al. Sonographic appearance of focal fatty infiltration of the pancreas. J. Clin. Ultrasound., Denver, v.38, n.1, p.45-47, 2010.
4. RICCl, C. et al. Noninvasive in vivo quantitative assessment of fat content in human liver. J. Hepatol., Amsterdam, v.27, n.1, p.108-113, 1997.

5. KÜHN, J. P. et al. Pancreatic steatosis demonstrated at $\mathrm{mr}$ imaging in the general population: clinical relevance. Radiology, Easton, v.276, n.1, p.129-135, 2015

6. KAWADA, N.; TANAKA, S. Elastography for the pancreas: current status and future perspective. World J. Gastroenterol., Pleasanton, v.22, n.12, p.3712-3724, 2016.

7. MELITAS, C.; MEISELMAN, M. Metabolic pancreatitis: pancreatic steatosis, hypertriglyceridemia, and associated chronic pancreatitis in 3 patients with metabolic syndrome. Case Rep. Gastroenterol., Switzerland, v.12, n.2, p.331-336, 2018.

8. CHOI, C.W. et al. World J Associated factors for a hyperechogenic pancreas on endoscopic ultrasound. World J. Gastroenterol., Pleasanton, v.16, n.34, p.4329-4334, 2010.

9. SEPE, P. S. et al. A prospective evaluation of fatty pancreas by using EUS. Gastrointest. Endosc., St Louis, v.73, n.5, p.987-993, 2011.

10. WONG, V.W. et al. Fatty pancreas, insulin resistance, and $\beta$-cell function: a population study using fat-water magnetic resonance imaging. Am. J. Gastroenterol., Philadelphia, v.109, n.4, p.589-597, 2014.

11. GULLO, L. et al. Can pancreatic steatosis explain the finding of pancreatic hyperenzymemia in subjects with dyslipidemia? Pancreas., Hagerstown, v.33, n.4, p.351-353, 2006.

12. LEE, J.S. et al. Clinical implications of fatty pancreas: correlations between fatty pancreas and metabolic syndrome. World J. Gastroenterol., Pleasanton, v.15, n.15, p.1869-1875, 2009.

13. GLASER, J.; STIENECKER, K. Pancreas and aging: a study using ultrasonography. Gerontology., New York, v.46, n.2, p.93-96, 2000.

14. NORONHA, M. et al. Alcohol and the pancreas. I. Clinical associations and histopathology of minimal pancreatic inflammation. Am. J. Gastroenterol., New York, v.76, n.2, p.114-119, 1981.

15. MIKOLASEVIC, I. et al. Nonalcoholic fatty liver and the severity of acute pancreatitis. Eur. J. Intern. Med., Basingstoke, v.38, p.73-78, 2017.

16. LINGVAY, I. et al. Noninvasive quantification of pancreatic fat in humans. J. Clin. Endocrinol. Metab., Springfield, v.94, n.10, p.40704076, 2009 .

17. ENGELMANN, G. et al. Feasibility study and control values of transient elastography in healthy children. Eur. J. Pediatr., Berlin, v.171, n.2, p.353-360, 2012.

18. LIM, S. et al. Differences in pancreatic volume, fat content, and fat density measured by multidetector-row computed tomography according to the duration of diabetes. Acta Diabetol., Berlin, v.51, n.5, p.739-748, 2014

19. SINGH, R.G. et al. Ectopic fat accumulation in the pancreas and its clinical relevance: A systematic review, meta-analysis, and meta-regression. Metabolism., Philadelphia, v.69, p.1-13, 2017.

Submetido em : 27/11/2019

Aceito em: 20/03/2020 